VoL. 44 (1991) [131-138]

\title{
REPRESENTATION OF TYPE A MONOIDS
}

\author{
U. ASIBONG-IBE
}

\begin{abstract}
A semigroup $T$ consisting of one-one mapping between certain principal left ideals in a type $A$ semigroup $S$ is constructed. $T$ is shown to be a type $A$ semigroup. A representation of $S$ by $T$ is then obtained which is analogous to Vagner-Preston's results on inverse semigroups.
\end{abstract}

\section{INTRODUCTION}

Many results are now available in the literature on type $A$ semigroups; some of which are analogous to those on inverse semigroups; see for example Fountain [6, 5], Asibong-Ibe [2, 3, 4], Amstrong [1] and Fountain and Lawson [7]. Because of the close relationship which exists between a type $A$ semigroup and an inverse semigroup, each type $A$ being basically a special type of subsemigroup of an inverse semigroup via an embedding, it is natural to ask whether a representation exists for a type $A$ semigroup similar to Vagner-Preston's for inverse semigroup. This paper answers this question.

Let us recall a few definitions. Let $S$ be a semigroup and $a, b \in S$. Then $(a, b) \in \mathcal{L}^{*}$ if and only if $a \mathcal{L} b$ is an oversemigroup of $S$. The relation $\mathcal{L}^{*}$ which properly contains the Green's relation $\mathcal{L}$ on $S$ has the following equivalent characterisation, see [10].

Lемма 1.1. Let $S$ be a semigroup and $a, b \in S$. The following are equivalent:

(i) $(a, b) \in \mathcal{L}^{*}$,

(ii) for all $x, y$ in $S$, $a x=a y$ if and only if $b x=b y$,

(iii) there exists an $S$-isomorphism $\lambda: a S^{1} \rightarrow b S^{1}$ such that $a \lambda=b$.

Lемма 1.2. Let $S$ be a semigroup and $e$ an idempotent in $S$. Then for any a in $S$, the following are equivalent:

(i) $(e, a) \in \mathcal{L}^{*}$,

(ii) $a e=a$, and for all $x, y$ in $S$, ax $=a y$ if and only if $e x=e y$.

$\mathcal{R}^{*}$ is dual to $\mathcal{L}^{*}$ and the above definition and properties of $\mathcal{L}^{*}$ apply in a dual manner to $\mathcal{R}^{*}$.

Received 23 August 1990

Copyright Clearance Centre, Inc. Serial-fee code: 0004-9729/91 \$A2.00+0.00. 
Let $S$ be a semigroup with a semilattice $E(S)$ of idempotents. Then $S$ is said to be an adequate semigroup if each $\mathcal{L}^{*}$-class and each $\mathcal{R}^{*}$-class contains an idempotent.

An adequate semigroup $S$ is said to be a type $A$ semigroup if for each $a$ in $S$ and $e$ in $E(S)$, ea $=a(e a)^{*}$ and $a e=(a e)^{+} a$, where $x^{*}$ and $x^{+}$are respectively idempotents in the $\mathcal{L}^{*}$ and $\mathcal{R}^{*}$ classes $L_{x}^{*}$ and $R_{x}^{*}$. A type $A$ semigroup has been characterised in the following way in [5].

Theorem 1.3. Let $S$ be an adequate semigroup. Then for $a \in S, e \in E(S)$, the following are equivalent:

(i) $S$ is a type $A$ semigroup,

(ii) $e S^{1} \cap a S^{1}=e a S^{1}$ and $S^{1} e \cap S^{1} a=S^{1} a e$, and

(iii) there exist embeddings $\lambda_{1}: S \rightarrow S_{1}$, and $\lambda_{2}: S \rightarrow S_{2}$ into inverse semigroups $S_{1}, S_{2}$ such that $a^{*} \lambda_{1}=\left(a \lambda_{1}\right)^{-1}\left(a \lambda_{1}\right)$ and $a^{+} \lambda_{2}=\left(a \lambda_{2}\right)\left(a \lambda_{2}\right)^{-1}$.

\section{TYPE $A$ SEMIGROUP OF MAPPINGS}

In this and subsequent sections, the term semigroup $S$ will refer to a type $A$ semigroup $S$ with $E(S)$ as its set of idempotents. Other notation used here agrees with that of [9] and [5].

Let $a \in S$; then $a^{+}, a^{*} \in E(S)$, and $a a^{*}=a^{+} a=a$. Consider the left principal ideals $S a^{+}$and $S a^{*}$ and let $x_{1} \in S a^{+}$. Then for some $x \in S, x_{1}=x a^{+} \in S a^{+}$ and $x_{1} a=x a^{+} a=x a=x a a^{*} \in S a^{*}$. Evidently for every $s$ in $S, s a a^{*}=s\left(a a^{*}\right)=$ $s a \in S a^{*}$. Let us define a mapping $\alpha_{a}: S a^{+} \rightarrow S a^{*}$ by putting for every $x$ in $S$, $x \alpha_{a}=x a$, where $a \in S$. Since $a a^{*}=a, S a=S a a^{*} \subseteq S a^{*}$, so for $x \in S, x a=$ $x a^{+} a=\left(x a^{+}\right) \alpha_{a} \in\left(S a^{+}\right) \alpha_{a}$ so evidently $\left(S a^{+}\right) \alpha_{a}=S a \subseteq S a^{*}$. Thus ran $\alpha_{a}=S a$. However, if $a$ is regular then $S a=S a^{*}$, thus in this case $\operatorname{ran} \alpha_{a}=S a^{*}$. Let us show that each $\alpha_{a}, a \in S$ is a one-to-one mapping.

Lemma 2.1. For each $a \in S, \alpha_{a}$ is a one-one mapping from $\mathrm{Sa}^{+}$into $\mathrm{Sa}^{*}$. Also $\alpha_{a}$ is onto if and only if $a$ is regular.

Proof: Consider the mapping $\alpha_{a}: S a^{+} \rightarrow S a^{*}$, and let $x a=y a$ for $x, y$ in $S$. Then $\left(x a^{+}\right) \alpha_{a}=x a=y a=\left(y a^{+}\right) \alpha_{a}$. But $a \mathcal{R}^{*} a^{+}$, so $x a=y a$ if and only if $x a^{+}=y a^{+}$for all $x, y$ in $S$. Consequently, $\alpha_{a}$ is a one-one mapping.

Now if $\alpha_{a}$ is onto then $\left(S a^{+}\right) \alpha_{a}=S a^{*}$. Thus $\left(S a^{+}\right) \alpha_{a}=S a^{+} a=S a=S a^{*}$; consequently $a \mathcal{L} a^{*}$, and $a$ must be regular. Conversely, if $a$ is regular, $a a^{-1} a=a$, $a^{*}=a^{-1} a$ and clearly $S a^{*}=S a$, so $\mathcal{L}_{a}$ is onto.

Corollary 2.2. For each $a \in S, \alpha_{a}$ has inverse $\alpha_{a-1}$ if and only if $a$ is regular.

ProOf: If $\alpha_{a}^{-1}=\alpha_{a^{-1}}$ then $x a^{+}=(x a) \alpha_{a}^{-1}=(x a) \alpha_{a^{-1}}=x a a^{-1}$. So $x a^{+} a=$ 
$x a a^{-1} a=x a$ and bijectivity of $\alpha_{a}$ forces $a^{+}=a a^{-1}$ so $a a^{-1} a=a$. Conversely if $a$ is regular $\alpha_{a}$ is bijective so $\alpha_{a}^{-1}$ exists and obviously $\alpha_{a}^{-1}=\alpha_{a-1}$.

Now let $a$ be a non-regular element in $S$. Let $\lambda: S a \rightarrow S a^{+}$be an $S$-system isomorphism with $a \lambda=a^{+}$. Thus given $\alpha_{a}: S a^{+} \rightarrow S a^{*}$ with $\operatorname{ran} \alpha_{a}=S a$ we can define $\alpha_{a}^{-1} \mid S a \rightarrow S a^{+}$by putting $\alpha_{a}^{-1}=\lambda$ so that $(x a) \alpha_{a}^{-1}=(x a) \lambda=x(a \lambda)=x a^{+}$ for $x \in S$. One checks that if $x \in S a^{+}, x \alpha_{a} \alpha_{a}^{-1}=(x a) \alpha_{a}^{-1}=x a^{+}=x$ and for each $y=x a$, we have $y \alpha_{a}^{-1} \alpha_{a}=x a^{+} \alpha_{a}=x a=y$. Observe that $S a \neq S a^{*}$ because an equality implies regularity of $a$, which is a contradiction to our assumption.

Now let us consider the subset $T$ of $\mathcal{I}(S)$, the symmetric inverse semigroup where $T=\left\{\alpha_{a} \mid a=S, \alpha_{a}: S a^{+} \rightarrow S a^{*}\right\}$ and impose the condition that $\alpha_{a}^{-1} \in T$ if and only if $\alpha_{a}^{-1}=\alpha_{a-1}$, that is if and only if $a$ is regular. Thus the domain and codomain of elements of $T$ are respectively the principal left ideals ganerated by $a^{+}$and $a^{*}$ for any $a \in S$.

An important fact is there is closure in $T$ with respect to the product of its elements. Let us show this as follows. Consider the mappings $\alpha_{a}: S^{+} \rightarrow S a^{*}$, $\alpha_{b}: S b^{+} \rightarrow S b^{*}$. Now $S a^{*} \cap S b^{+}=S a^{*} b^{+}$, and $a^{*} b^{+}=\left(a b^{+}\right)^{*}$. Consequently $a b^{+}=a a^{*} b^{+}=a\left(a b^{+}\right)^{*} ;$ hence $a\left(a b^{+}\right)^{*}=a b^{+}=\left(a b^{+}\right)^{+} a$. Since $S a \subseteq S a^{*}$ then $S a \cap S b^{+}=S a b^{+} \subseteq S a^{*} b^{+}$so that $S a b^{+}=S\left(a b^{+}\right)^{+} a=S(a b)^{+} a=S(a b)^{+} \alpha_{a}$. But $S a b^{+} \subseteq S b^{+}$, and hence $\left(S a b^{+}\right) \alpha_{b} \subseteq\left(S b^{+}\right) \alpha_{b}$, and $\left(S a b^{+}\right) \alpha_{b}=S(a b)^{+} \alpha_{a} \alpha_{b}=S a b$. Indeed, since $\left(S a^{*} b^{+}\right) \alpha_{b}=S a^{*} b$ and $a^{*} b=b\left(a^{*} b\right)^{*}=b(a b)^{*}$, one checks that $S a^{*} b=S b(a b)^{*} \subseteq S(a b)^{*}$. With $S a b \subseteq S(a b)^{*}$, it is clear that the codomain of $\alpha_{a} \alpha_{b}$ is $S(a b)^{*}$ and its domain is $S(a b)^{+}$. Evidently, it follows from these facts that $\alpha_{a} \alpha_{b}=\alpha_{a b}$, showing closure property in $T$. It is then clear that $T$ is a semigroup.

Let $a, b$ be regular elements in $S$. Then $(a b)$ is regular with inverse $(a b)^{-1} \in S$. Also $\alpha_{a}, \alpha_{b}$ are regular in $T$ and evidently $\alpha_{a b}=\alpha_{a} \alpha_{b}$ is regular in $T$ with inverse $\alpha_{(a b)^{-1}} \in T, \alpha_{a b}^{-1}=\left(\alpha_{a} \alpha_{b}\right)^{-1}=\alpha_{b}^{-1} \alpha_{a}^{-1}=\alpha_{b-1} \alpha_{a-1}=\alpha_{b^{-1} a^{-1}}=\alpha_{(a b)^{-1}} \in T$. Let us now show below that $T$ is a type $A$ monoid.

TheOREM 2.3. For a type $A$ semigroup $S$, the set $T=\left\{\alpha_{a} \mid a \in S, \alpha_{a}: S a^{+} \rightarrow\right.$ $\left.S a^{*}\right\}$ such that for each $x$ in $S, x \alpha_{a}=x a$, is a type $A$ monoid.

We will prove this fact through the following lemmas.

LEMMA 2.4.

(i) $\left(\alpha_{a}, \alpha_{b}\right) \in \mathcal{L}^{*}(T)$ if and only if $(a, b) \in \mathcal{L}^{*}(S)$, and

(ii) $\left(\alpha_{a}, \alpha_{b}\right) \in \mathcal{R}^{*}(T)$ if and only if $(a, b) \in \mathcal{R}^{*}(S)$.

Proof: Let $\left(\alpha_{a}, \alpha_{b}\right) \in \mathcal{L}^{*}$ for $\alpha_{a}, \alpha_{b}$ in $T$. Then for all $\alpha_{c}, \alpha_{d}$ in $T$ we have that

$$
\alpha_{a} \alpha_{c}=\alpha_{a} \alpha_{d} \text { if and only if } \alpha_{b} \alpha_{c}=\alpha_{b} \alpha_{d}
$$


Let $\alpha_{a} \alpha_{c}=\alpha_{a} \alpha_{d}$. Then $\left(\operatorname{dom} \alpha_{a} \alpha_{c}\right) \alpha_{a}=\operatorname{ran} \alpha_{a} \cap \operatorname{dom} \alpha_{c}=\left(\operatorname{ran} \alpha_{a} \cap \operatorname{dom} \alpha_{d}\right)=$ $\left(\operatorname{dom} \alpha_{a} \alpha_{d}\right) \alpha_{a}$. Also $\left(\operatorname{ran} \alpha_{a} \cap \operatorname{dom} \alpha_{c}\right) \alpha_{c}=\left(\operatorname{ran} \alpha_{a} \cap \operatorname{dom} \alpha_{d}\right) \alpha_{d}$. Now if $x \alpha_{a} \in$ $\operatorname{ran} \alpha_{a} \cap \operatorname{dom} \alpha_{c}$, then the equality $\operatorname{ran} \alpha_{a} \cap \operatorname{dom} \alpha_{c}=\operatorname{ran} \alpha_{a} \cap \operatorname{dom} \alpha_{d}$ implies that for all $x$ in $S, x a \alpha_{c}=x a \alpha_{d}$. That is, $x a c=x a d$ and in particular for $x=a^{+}$, $a c=a^{+} \alpha_{a c}=a^{+} a c=a^{+} a d=a^{+} \alpha_{a d}=a d$. Thus if $\alpha_{a c}=\alpha_{a d}$ then $a c=a d$ for any $\alpha_{a c}, \alpha_{a d} \in T$. But $\alpha_{a c}=\alpha_{a d}$ if and only if $\alpha_{b c}=\alpha_{b d}$. It can be shown that whenever this holds then $a c=a d$ if and only if $b c=b d$. Hence $(a, b) \in \mathcal{L}^{*}(S)$. Conversely, let $a c=a d$. Then $\alpha_{a c}=\alpha_{a d}$ and so $\alpha_{a} \alpha_{c}=\alpha_{a} \alpha_{d}$. But for all $c, d \in S, a c=a d$ implies $b c=b d$ and whenever $a c=a d$, then $\alpha_{a} \alpha_{c}=\alpha_{a} \alpha_{d}$. Hence for all $c, d \in S$, we can deduce that $\alpha_{a} \alpha_{c}=\alpha_{a} \alpha_{d}$ implies $\alpha_{b} \alpha_{c}=\alpha_{b} \alpha_{d}$. Since this is true for all $\alpha_{c}, \alpha_{d} \in T$, then $\left(\alpha_{a}, \alpha_{b}\right) \in \mathcal{L}^{*}(T)$, which completes the proof of (i). The proof of (ii) is similar, so the lemma is proved.

From the above lemma we have the following.

Corollary 2.5. Let $\alpha_{a}, \alpha_{b} \in T$. Then

(i) $\left(\alpha_{a}, \alpha_{b}\right) \in \mathcal{H}^{*}(T)$ if and only if $(a, b) \in \mathcal{H}^{*}(S)$,

(ii) $\left(\alpha_{a}, \alpha_{b}\right) \in \mathcal{D}^{*}(T)$ if and only if $(a, b) \in \mathcal{D}^{*}(S)$.

Proof: (i) If $\left(\alpha_{a}, \alpha_{b}\right) \in \mathcal{H}^{*}(T)$, then obviously $\left(\alpha_{a}, \alpha_{b}\right) \in \mathcal{L}^{*}(T)$ and $\left(\alpha_{a}, \alpha_{b}\right) \in$ $\mathcal{R}^{*}(T)$ and by Lemma $2.4(a, b) \in \mathcal{L}^{*} \cap \mathcal{R}^{*}=\mathcal{H}^{*}$. Conversely, if $(a, b) \in \mathcal{H}^{*}$, then $\left(\alpha_{a}, \alpha_{b}\right) \in \mathcal{H}^{*}(T)$ holds from Lemma 2.4 .

(ii) For $\left(\alpha_{a}, \alpha_{b}\right) \in \mathcal{D}^{*}(T)$, there exist $\alpha_{z_{1}} \alpha_{z_{2}}, \ldots, \alpha_{z_{n}} \in T$ such that

$$
\alpha_{a} \mathcal{L}^{*} \alpha_{x_{1}} \mathcal{R}^{*} \alpha_{z_{3}} \mathcal{L}^{*} \ldots \alpha_{x_{n}} \mathcal{R}^{*} \alpha_{b}
$$

But Lemma 2.4 implies that in $S, a \mathcal{L}^{*} x_{1} \mathcal{R}^{*} x_{2} \mathcal{L}^{*}, \ldots, x_{n} \mathcal{R} b$ whence $(a, b) \in \mathcal{D}^{*}$. The converse can also be shown using Lemma 2.4 .

To identify idempotent elements in $T$, observe that if $a$ in $S$ is an idempotent then $a^{+}=a^{*}=a$. If $x \in S e, x e=x$ so that $x \alpha_{e}=x e, \alpha_{e}=1_{S e}$.

LEMma 2.6. An element $\alpha_{a} \in T$ is an idempotent if and only if $a$ in $S$ is an idempotent. Moreover, $E(T)$ is a semilattice.

Proof: If $\alpha_{a}$ is an idempotent then $\alpha_{a}^{2}=\alpha_{a}$ implies dom $\alpha_{a}^{2}$ $=\left(\operatorname{ran} \alpha_{a} \cap \operatorname{dom} \alpha_{a}\right) \alpha_{a}^{-1}=\operatorname{dom} \alpha_{n}$, that is, $\operatorname{ran} \alpha_{a} \cap \operatorname{dom} \alpha_{a}=\operatorname{ran} \alpha_{a}$ so that $\operatorname{ran} \alpha_{a} \subseteq$ $\operatorname{dom} \alpha_{a}$. Also $\operatorname{ran} \alpha_{a}^{2}=\left(\operatorname{ran} \alpha_{a} \cap \operatorname{dom} \alpha_{a}\right) \alpha_{a}=\operatorname{ran} \alpha_{a}$ hence $\operatorname{dom} \alpha_{a}, \subseteq \operatorname{ran} \alpha_{a}$. From both inclusions, $\operatorname{dom} \alpha_{a}=\tan \alpha_{a}$. Thus $S a=S a^{+}$and for $x \in \operatorname{dom} \alpha_{a}, x \alpha_{a}^{2}=x \alpha_{a}$, that is $x a^{2}=x a$, so in particular, for $x=a^{+}, a^{2}=a^{+} a^{2}=a^{+} a=a$. Therefore $a$ is an idempotent in $S$.

Conversely if $a$ is an idempotent in $S$ then $a^{*}=a^{+}$so that $\mathrm{Sa}^{+}=\mathrm{Sa}^{*}$ and quite clearly $\operatorname{dom} \alpha_{a}^{2}=\operatorname{dom} \alpha_{a}=\operatorname{ran} \alpha_{a}=\operatorname{ran} \alpha_{a}^{2}$ and for all $x \in S a^{+}, x a^{2}=x a$. Hence for all $x \in S a^{+}, x \alpha_{a}^{2}=x \alpha_{a}$, so $\alpha_{a}^{2}=\alpha_{a}$. 
Let $\alpha_{e}, \alpha_{f} \in E(T)$, the set of idempotents of $T$. Now $\alpha_{e} \alpha_{f}=\alpha_{e f}=\alpha_{f e}=\alpha_{f} \alpha_{e}$ and if $e \leqslant f$, ef $=f e=e$, so $\alpha_{e} \alpha_{f}=\alpha_{f} \alpha_{e}=\alpha_{e}$. This completes the proof of the lemma.

For $a \in S, a^{*} \in L_{a}^{*}, a^{+} \in R_{a}^{*}$ and $\alpha_{a} \alpha_{a^{*}}=\alpha_{a a^{*}}=\alpha_{a}$ and $\alpha_{a}+\alpha_{a}=\alpha_{a}+a=\alpha_{a}$. Evidently $\left(\alpha_{a}, \alpha_{a^{*}}\right) \in \mathcal{L}^{*}(T)$ by Lemma 2.4, so we have

LEMMA 2.7. For each $\alpha_{a} \in T$

(i) $\left(\alpha_{a}, \alpha_{a}\right) \in \mathcal{L}^{*}(T)$ and

(ii) $\left(\alpha_{a}, \alpha_{a}+\right) \in \mathcal{R}^{*}(T)$.

Let $L_{\alpha_{a}}^{*}$ and $R_{\alpha_{a}}^{*}$ be the $\mathcal{L}^{*}(T)$ and $\mathcal{R}^{*}(T)$ classes containing $\alpha_{a}$. Let us denote by $\alpha_{a}^{*}$ and $\alpha_{a}^{+}$the unique idempotents in $L_{\alpha_{a}}^{*}$ and $R_{\alpha_{a}}^{*}$ respectively. Now for $a \in S$, $e \in E(S), e a=a(e a)^{*}, a e=(a e)^{+} a$, and consequently $\alpha_{e} \alpha_{a}=\alpha_{e a}=\alpha_{a(e a)^{*}}=$ $\alpha_{a} \alpha_{(e a)^{*}}=\alpha_{a} \alpha_{e a}^{*}=\alpha_{a}\left(\alpha_{e} \alpha_{a}\right)^{*}$ and similarly $\alpha_{a} \alpha_{e}=\left(\alpha_{a} \alpha_{e}\right)^{+} \alpha_{a}$. Thus we have proved that

LEMMA 2.8. For $\alpha_{a}, \alpha_{e} \in T$,

(i) $\alpha_{e} \alpha_{a}=\alpha_{a}\left(\alpha_{e} \alpha_{a}\right)^{*}$ and

(ii) $\alpha_{a} \alpha_{e}=\left(\alpha_{a} \alpha_{e}\right)^{+} \alpha_{a}$.

These last observations together with Lemmas 2.4 to 2.7 complete the proof of Theorem 2.3.

Let $\beta_{a}: a^{*} S \rightarrow a^{+} S, a \in S$ where $x \beta_{a}=a x$ for $x \in S$; using methods similar to the above, $\beta_{a}$ is a one-to-one mapping satisfying Lemmas 2.4 to 2.8 and

Corollary 2.9. $T^{*}=\left\{\beta_{a} \mid a \in S\right\}$ is a type $A$ semigroup.

\section{Representation of tYPE $A$ monoid}

We show here that there is a Vagner-Preston type representation from a type $A$ semigroup $S$ into a type $A$ semigroup of mappings on a set $X$. Let $X=S, a \in S$, and let $\varphi: S \rightarrow T$ be a mapping such that $a \varphi=\alpha_{a}$, where $T=\left\{\alpha_{a} \mid a \in S\right\}$ is the type $A$ semigroup in Theorem 2.3 above.

THEOREM 3.1. The mapping $\varphi: S \rightarrow T$, where $a \varphi=\alpha_{a}$, is an isomorphism from $S$ onto $T$.

Proof: If $a, b \in S$, then $(a b) \varphi=\alpha_{a b}=\alpha_{a} \alpha_{b}=a \varphi$.b $b$. Also $a \varphi=b \varphi$ implies $\alpha_{a}=\alpha_{b}$, which in turn implies that $S a^{+}=S b^{+}, S a=S b$, the domains and ranges of $\alpha_{a}$ and $\alpha_{b}$, respectively, and for all $x \in S a^{+}, x \alpha_{a}=x \alpha_{b}$. Now $S a^{+}=S b^{+}$implies $a^{+} \mathcal{L} b^{+}$and hence $a^{+}=b^{+}$. Similarly $S a^{*}=S b^{*}$ implies $a^{*}=b^{*}$. But $x \alpha_{a}=x \alpha_{b}$ implies that $x a=x b$ for all $x \in S a^{+}$; hence for $x=a^{+}, a=a^{+} a=a^{+} b=b^{+} b=b$. Thus if $\alpha_{a}=\alpha_{b}$ then $a=b$, showing that $\varphi$ is a one-to-one homomorphism. By definition of $T, \varphi$ is onto, so the proof is complete. 
From Corollary 2.9, $T^{\prime}=\left\{\beta_{a} \mid a \in S\right\}$ in type $A$ semigroup and so

Corollary 3.2. Let $\psi: S \rightarrow T^{\prime}$ be a mapping given by $a \psi=\beta_{a}$, for $a \in S$. Then $\psi$ is an isomorphism.

Proof: As in Theorem 3.1 above, $(a b) \psi=\beta_{a b}=\beta_{a} \beta_{b}=(a \psi)(b \psi)$, so $\psi$ is a one-to-one homomorphism from $S$ onto $T^{\prime}$. This completes the proof.

Let $S$ be a left type $A$ monoid and $T=\left\{\alpha_{a} \mid a \in S_{\gamma} \alpha_{a}: S a^{+} \rightarrow S a^{*}\right\}$ where $\alpha_{a}^{-1} \in T$ if and only if $\alpha_{a}^{-1}=\alpha_{a-1}$, that is, if and only if $a$ is regular.

THEOREM 3.3. $T$ is a left adequate semigroup.

Proof: Consider $\alpha_{a}: S a^{+} \rightarrow S a^{*}, \alpha_{b}: S b^{+} \rightarrow S b^{*}$ as defined earlier, where $a, b \in S$ are non-regular. Now $\operatorname{ran} \alpha_{a}=S a \neq S a^{*}$ and $\operatorname{ran} \alpha_{a} \cap \operatorname{dom} \alpha_{b}=S a \cap$ $S b^{+}=S a b^{+}=S(a b)^{+} a=\left(\operatorname{dom} \alpha_{a b}\right) \alpha_{a}$. Also $\left(\operatorname{ran} \alpha_{a} \cap \operatorname{dom} \alpha_{b}\right) \alpha_{b}=S a b^{+} b=S a b \subseteq$ $S(a b)^{*}$, so that $\left(\operatorname{ran} \alpha_{a} \cap \operatorname{dom} \alpha_{b}\right) \alpha_{b}=\operatorname{ran} \alpha_{a b}$. Since $\left(\operatorname{dom} \alpha_{a b}\right) \alpha_{a} \alpha_{b}=\operatorname{ran} \alpha_{a b}$, by the previous lemma, $T$ is a semigroup.

The proof of the theorem is complete by noting that the relevant aspects of Lemmas $2.4-2.7$ above hold for $T$ as well.

In fact $T$ is a left type $A$ semigroup since for $\alpha_{a}, \alpha_{e} \in T, \alpha_{a} \alpha_{e}=\left(\alpha_{a} \alpha_{e}\right)^{+} \alpha_{a}$, which is true by Lemma 2.8 since as $S$ is a left type $A$ monoid, for $a$ in $S, e \in E(S)$, $a e=(a e)^{+} a$.

Since ea $\neq a(e a)^{*}$ does not hold in general for a left type $A$ semigroup $S$ with $a \in S$, and $e$ an idempotent, in general the equality $\alpha_{e} \alpha_{a}=\alpha_{a}\left(\alpha_{e} \alpha_{a}\right)^{*}$ does not hold. However, we show below an example in which $S$ is left type $A$ and $T$ a type $A$ monoid.

EXAMPLE: Consider the semigroup $S$ with the following multiplication table:

\begin{tabular}{l|lllll}
$\cdot$ & $\boldsymbol{e}$ & $\boldsymbol{f}$ & $\boldsymbol{z}$ & $\boldsymbol{a}$ & $\boldsymbol{c}$ \\
\hline $\boldsymbol{e}$ & $\boldsymbol{e}$ & $\boldsymbol{z}$ & $\boldsymbol{z}$ & $\boldsymbol{c}$ & $\boldsymbol{c}$ \\
$\boldsymbol{f}$ & $\boldsymbol{z}$ & $\boldsymbol{f}$ & $\boldsymbol{z}$ & $z$ & $z$ \\
$z$ & $z$ & $z$ & $z$ & $z$ & $z$ \\
$\boldsymbol{a}$ & $z$ & $a$ & $z$ & $z$ & $z$ \\
$\boldsymbol{S}$ & $\boldsymbol{z}$ & $\boldsymbol{a}$ & $\boldsymbol{z}$ & $\boldsymbol{z}$ & $\boldsymbol{z}$
\end{tabular}

The $\mathcal{L}^{*}$ classes of $S$ are $\{f, a, c\},\{z\},\{e\}$ and the $\mathcal{R}^{*}$ classes are $\{e, a, c\},\{f\},\{z\}$. It is easy to check that for each idempotent $u \in E(S)$ and each $x \in S, x u=(x u)^{+} x$, and that $c=e a \neq a(e a)^{*}=a f=a$, hence $S$ is left type $A$ but not a right type $A$ monoid.

Now define $\alpha_{a}: S a^{+} \rightarrow S a^{*}$ as usual. So $T=\left\{\alpha_{e}, \alpha_{f}, \alpha_{z}, \alpha_{a}, \alpha_{c}\right\}$, with $\mathcal{L}^{*}$ classes $\left\{\alpha_{a}, \alpha_{c}, \alpha_{f}\right\},\left\{\alpha_{e}\right\},\left\{\alpha_{z}\right\}$ and $\mathcal{R}^{*}$-classes: $\left\{\alpha_{a}, \alpha_{c}, \alpha_{e}\right\},\left\{\alpha_{f}\right\}$ and $\left\{\alpha_{z}\right\}$. It is straightforward to verify that $\alpha_{e}, \alpha_{f}$ are the only elements with $\alpha_{e}^{-1}=\alpha_{e-1}=\alpha_{e}$, $\alpha_{f}^{-1}=\alpha_{f^{-1}}=\alpha_{f}$ so $\alpha_{e^{-1}}, \alpha_{f^{-1}} \in T$. Now for all $u \in\{e, f\}, x \in S$, 
$\alpha_{z} \alpha_{u}=\left(\alpha_{x} \alpha_{u}\right)^{+} \alpha_{x}$ but while ea $\neq a(e a)^{*}$, we have $\alpha_{e} \alpha_{a}=\alpha_{e a}=\alpha_{c}$, and $\alpha_{a} \alpha_{(e a)^{*}}=\alpha_{a}\left(\alpha_{e} \alpha_{a}\right)^{*}=\alpha_{a} \alpha_{c}^{*}=\alpha_{a} \alpha_{f}=\alpha_{a}$ and for all $x \in S, x \alpha_{c}=x \alpha_{a}$, hence $\alpha_{c}=\alpha_{a}$, since $S c^{+}=S a^{+}$, and $S c=S a$. One also finds that $\alpha_{e} \alpha_{c}=\alpha_{c}\left(\alpha_{e} \alpha_{c}\right)^{*}$, and in general $\alpha_{u} \alpha_{x}=\alpha_{x}\left(\alpha_{y} \alpha_{x}\right)^{*}$ so $T$ is a type $A$, with $E=\left\{\alpha_{e}, \alpha_{f}, \alpha_{z}\right\}$ as a semilattice.

From all the forgoing we have for the left type $A$ semigroup in the table:

THEOREM 3.4. $S$ is isomorphic to a left type $A$ semigroup of one-to-one mappings on $S$.

Let us consider an arbitrary left type $A$ semigroup $S$ and $T$, the semigroup of one-to-one mappings $\alpha_{a}, a \in S$. The following result holds.

TheOREM 3.5. Let $S$ be a left type $A$ semigroup; then $T$ is a left type $A$ semigroup. Moreover $S$ is isomorphic to $T$.

To see this clearly, consider an arbitrary left type $A$ monoid $S$ and $T=\left\{\alpha_{a} \mid a \in\right.$ $\left.S, \alpha_{a}: S a^{+} \rightarrow S a^{*}\right\}$ where $\alpha_{a}: S a^{+} \rightarrow S a^{*}$ is defined by putting

$$
x \alpha_{a}=x a, \text { for every } x \text { in } S,
$$

and $\alpha_{a}^{-1} \in T, a \in S$ if and only if $\alpha_{a}^{-1}=\alpha_{a^{-1}}$. Then $S a \cap S b^{+}=S a b^{+}$, for $a \in S$, $b^{+} \in E(S)$, and if $\alpha_{a}: S a^{+} \rightarrow S a^{*}, \alpha_{b}: S b^{+} \rightarrow S b^{*}$ and $a, b \in S$ have no inverses in $S, \operatorname{ran} \alpha_{a}=S a \neq S a^{*}, \operatorname{ran} \alpha_{b}=S b \neq S b^{*}$. Also $\operatorname{dom} \alpha_{a} \alpha_{b}=S(a b)^{+}=\operatorname{dom} \alpha_{a b}$ and $\operatorname{ran} \alpha_{a} \alpha_{b}=S a b=\operatorname{ran} \alpha_{a b}$ and $T$ is a semigroup.

$S a=S a^{*}$ if and only if $S$ is regular and in such cases $\alpha_{a}$ is bijective and $\alpha_{a}^{-1}=$ $\alpha_{a-1}$.

That $T$ is a left type $A$ semigroup is shown in Theorem 3.3 together with Lemmas $2.4-2.6$ and the following lemmas.

LEMma 3.6. $\left(\alpha_{a}, \alpha_{a^{+}}\right) \in \mathcal{R}^{*}(T)$ for all $a \in S, a^{+} \in E(S)$.

LEMMA 3.7. $\alpha_{a} \alpha_{e}=\left(\alpha_{a} \alpha_{e}\right)^{+} \alpha_{a}$ for all $a \in S, e \in E(S)$.

PROOF: $\alpha_{a} \alpha_{e}=\alpha_{a e}=\alpha_{(a e)^{+} a}=\alpha_{(a e)}+\alpha_{a}=\alpha_{a e}^{+} \alpha_{a}=\left(\alpha_{a} \alpha_{e}\right)^{+} \alpha_{a}$, since $a e=$ $(a e)^{+} a$.

The proof of Theorem 3.5 is complete by noting that if $\psi: S \rightarrow T$ is a mapping where $\psi$ is defined by $a \psi=\alpha_{a}$ for $a \in S$, then for all $a, b$ in $S$

$$
(a b) \psi=(a \psi)(b \psi)
$$

and $\psi$ is one-to-one and onto.

If $S$ is an adequate semigroup which is not type $A$, the above result may not hold. Now for $a, b \in S$ suppose that $z \in S a \cap S b^{+}$. Then $z=s a=t b^{+}$for some $s, t \in S$ 
and since $z=a b^{+}=s a b^{+} \in S a b^{+}$then $S a \cap S b^{+} \subseteq S a b^{+}$. To understand the situation clearly, let $S=C \cup D \cup\{1\}$ where $C=\langle a\rangle$ is the free semigroup on $a$ and $D=\langle b\rangle$ the free monoid generated by $b$, with multiplication in $S$ defined by $a^{m} b^{n}=b^{m+n}$, $b^{m} a^{m}=a^{m+n}$, for $m>0, n \geqslant 0, b^{0}=c$, and 1 is the identity in $S$. The $\mathcal{L}^{*}$ - and $\mathcal{R}^{*}$-classes of $S$ are respectively $C \cup\{1\}, D$ and $\{1\}, C \cup D$. For $a, b \in S, a^{*}=1$, $a^{+}=e, b^{*}=b^{+}=e, S a \cap S b^{+}=\emptyset, S a b^{+}=D \backslash\{e\}$, so $S a \cap S b^{+} \neq S a b^{+}$. Moreover, $\alpha_{a}: S a^{+} \rightarrow S a^{*}$ is not one-to-one since for $x=a^{t}, y=b^{t}, x \alpha_{a}=y \alpha_{a}$ but $x \neq y$.

\section{REFERENCES}

[1] S. Amstrong, 'The structure of type $A$ semigroup', Semigroup Forum 29 (1984), 319-336.

[2] U. Asibong-Ibe, Structure of Type A Semigroups, D. Phil. Thesis (University of York, 1981).

[3] U. Asibong-Ibe, '*-bisimple type $A$ w-semigroup', Semigroup Forum 31 (1985), 99-117.

[4] U. Asibong-Ibe, '*-simple type $A$ w-semigroup' (to appear).

[5] J.B. Fountain, 'Adequate semigroups', Proc. Edinburgh Math. Soc. 22 (1979), 110-125.

[6] J.B. Fountain, 'A class of right PP monoids', Quart. J. Math. Oxford 28 (1977), 285-300.

[7] J.B. Fountain and Lawson, 'Translational hull of adequate semigroups', Semigroup Forum 32 (1985), 79-86.

[8] J.M. Howie, 'An introduction to semigroup theory', in London Math. Soc. Monographs 7 (Academic Press, 1976).

[9] D.B. McAlister, 'One-to-one partial translations of right cancellative semigroups', J. Algebra 45 (1976), 231-251.

[10] W.D. Munn, 'Regular w-semigroups', Glasgow Math. J. 9 (1968), 46-66.

Department of Mathematics University of Port Harcourt

Port Harcourt

Nigeria 